University of Wollongong

Research Online

Faculty of Engineering and Information

Faculty of Engineering and Information

Sciences - Papers: Part A

Sciences

$1-1-2014$

Concept and simulation study of a novel localization method for robotic endoscopic capsules using multiple positron emission markers

Trung Duc Than

University of Wollongong, dtt581@uowmail.edu.au

Gursel Alici

University of Wollongong, gursel@uow.edu.au

Steven Harvey

Wollongong Hospital, sharvey@uow.edu.au

Hao Zhou

University of Wollongong, hz467@uowmail.edu.au

Weihua Li

University of Wollongong, weihuali@uow.edu.au

Follow this and additional works at: https://ro.uow.edu.au/eispapers

Part of the Engineering Commons, and the Science and Technology Studies Commons

Research Online is the open access institutional repository for the University of Wollongong. For further information contact the UOW Library: research-pubs@uow.edu.au 


\title{
Concept and simulation study of a novel localization method for robotic endoscopic capsules using multiple positron emission markers
}

\begin{abstract}
Purpose: Over the last decade, wireless capsule endoscope has been the tool of choice for noninvasive inspection of the gastrointestinal tract, especially in the small intestine. However, the latest clinical products have not been equipped with a sufficiently accurate localization system which makes it difficult to determine the location of intestinal abnormalities, and to apply follow-up interventions such as biopsy or drug delivery. In this paper, the authors present a novel localization method based on tracking three positron emission markers embedded inside an endoscopic capsule. Methods: Three spherical 22Na markers with diameters of less than $1 \mathrm{~mm}$ are embedded in the cover of the capsule. Gamma ray detectors are arranged around a patient body to detect coincidence gamma rays emitted from the three markers. The position of each marker can then be estimated using the collected data by the authors' tracking algorithm which consists of four consecutive steps: a method to remove corrupted data, an initialization method, a clustering method based on the Fuzzy C-means clustering algorithm, and a failure prediction method. Results: The tracking algorithm has been implemented in MATLAB utilizing simulation data generated from the Geant4 Application for Emission Tomography toolkit. The results show that this localization method can achieve real-time tracking with an average position error of less than $0.4 \mathrm{~mm}$ and an average orientation error of less than $2^{\circ}$. Conclusions: The authors conclude that this study has proven the feasibility and potential of the proposed technique in effectively determining the position and orientation of a robotic endoscopic capsule.
\end{abstract}

\section{Keywords}

markers, method, robotic, endoscopic, capsules, multiple, positron, concept, simulation, study, novel, emission, localization

\section{Disciplines}

Engineering | Science and Technology Studies

\section{Publication Details}

Than, T. Duc., Alici, G., Harvey, S., Zhou, H. \& Li, W. (2014). Concept and simulation study of a novel localization method for robotic endoscopic capsules using multiple positron emission markers. Medical Physics, 41 (7), 072501-1-072501-14. 
Concept and simulation study of a novel localization method for robotic endoscopic capsules using multiple positron emission markers ${ }^{\mathrm{a})}$

Trung Duc Than ${ }^{\mathrm{b})}$ and Gursel Alici ${ }^{\mathrm{c})}$

School of Mechanical, Materials and Mechatronic Engineering,

5

University of Wollongong, New South Wales, 2522, Australia

Steven Harvey ${ }^{\mathrm{d})}$

Department of Nuclear Medicine, Wollongong Hospital, New South Wales, 2500, Australia

Hao Zhou ${ }^{\mathrm{e})}$ and Weihua $\mathrm{Li}^{\mathrm{f}}{ }^{\mathrm{f}}$

School of Mechanical, Materials and Mechatronic Engineering,

University of Wollongong, New South Wales, 2522, Australia

(Dated: 27 February 2014) 
Purpose: Over the last decade, wireless capsule endoscope has been the tool of choice for non-invasive inspection of the gastrointestinal tract, especially in the small intestine. However, the latest clinical products have not been equipped with a sufficiently accurate localization system which makes it difficult to determine the location of intestinal abnormalities, and to apply follow-up interventions such as biopsy or drug delivery. In this paper, we present a novel localization method based on tracking three positron emission markers embedded inside an endoscopic capsule.

Methods: Three spherical ${ }^{22} \mathrm{Na}$ markers with diameters of less than $1 \mathrm{~mm}$ are embedded in the cover of the capsule. Gamma ray detectors are arranged around a patient body to detect coincidence gamma rays emitted from the three markers. The position of each marker can then be estimated using the collected data by our tracking algorithm which consists of four consecutive steps: a method to remove corrupted data, an initialization method, a clustering method based on the Fuzzy C-means clustering algorithm, and a failure prediction method.

Results: The tracking algorithm has been implemented in Matlab utilizing simulation data generated from the GATE (Geant4 Application for Emission Tomography) toolkit. The results show that this localization method can achieve real-time tracking with an average position error of less than $0.4 \mathrm{~mm}$ and an average orientation error of less than $2^{\circ}$.

Conclusions: We conclude that this study has proven the feasibility and potential of the proposed technique in effectively determining the position and orientation of a robotic endoscopic capsule. 


\section{INTRODUCTION}

A Wireless Capsule Endoscope (WCE), being a non-invasive and well-tolerated diagnostic tool, has been a preferred choice to investigate abnormalities in the Gastrointestinal (GI) tract since its introduction in $2000^{1}$. Over a million of examinations using WCE have been performed all over the world ${ }^{2}$. Although many patients have benefited from these examinations, deaths caused by GI diseases and disorders are still at a high rate. It was reported by the World Health Organization that there were more than 1.4 million deaths caused by stomach and colorectal cancer in $2009^{3}$. The American Cancer Society also reports that approximately 50,000 people die each year in the United States due to colorectal cancer ${ }^{4}$. Additionally, other site-specific GI diseases also impose substantial health burden on the society. Therefore, the next generation of WCE is desired to have not only the diagnostic functionality, but also the therapeutic capabilities such as biopsy, targeted drug delivery, and micro surgery ${ }^{5-7}$.

A major issue that constrains the progress to develop a fully robotic WCE is that it has not been equipped with an accurate localization system to provide both position and orientation information of the capsule. With the latest version of WCE, the endoscopists are able to detect pathologies by reviewing the clear images of abnormalities in the GI tract. However, they are presently unable to locate the abnormalities accurately which, to some extent, limits the effectiveness of the diagnosis. One of the important information that the endoscopists need during the diagnosis is the distance that the capsule has travelled along the GI tract. It would be possible to estimate this information and reconstruct the movement trajectory if the capsule is tracked continuously in real-time. Furthermore, in some cases, the endoscopists may want to return to the sites of interest for further diagnosis or followup interventions. Without accurate localization data, it is very difficult to accomplish this capability.

Another limitation of the current commercial endoscopic capsules is their passive movement driven by natural peristalsis of the GI tract. Endowing the capsule with an active actuation system would speed up the endoscopic examination and minimize the possibility of missing symptoms. It would also greatly reduce the risks of retention ${ }^{8}$. However, an active movement control for the capsule would not be accomplished without prompt feedback provided by a localization system. This again emphasizes the significance of solving 
the problem of localization as visual feedback via endoscopic images is not sufficient. For example, one of the most popular methods for controlling the movement of the capsule is to use an external magnetic field to move or rotate an internal permanent magnet integrated inside the capsule ${ }^{9,10}$. In this method, the position and orientation data are essential to align or maintain stable link between the external magnetic field and the internal magnetic field; and to control the strength and direction of the external magnetic field.

A variety of localization methods for WCE have been proposed in the literature ${ }^{11}$. The first idea was to utilize radio-frequency signals emitted from a capsule for transmission of endoscopic images ${ }^{12}$. The signal strength of the received signals measured at eight antennas placed on the patient abdomen was employed to estimate the position of the capsule. One advantage of this technique is that it does not occupy any additional space or consume any power of the capsule. However, the method is limited to $2 \mathrm{D}$ tracking with very low spatial resolution (an average position error of $37.7 \mathrm{~mm}$ ).

Another popular technique is through tracking a permanent magnet embedded inside a capsule. A magnetic sensor array is built around the patient's body to measure the magnetic flux intensities. Based on the mathematical relationship between the strength and the direction of the magnetic field and the position and orientation of the magnet, the localization parameters of the capsule can be computed by solving inverse equations. Although this method can achieve an orientation error of $1.6^{\circ}$ and an average position error of $1.8 \mathrm{~mm}^{13}$, the method is not compatible with the presence of magnetic actuation systems due to an interference between the two applied magnetic fields.

Several research efforts have been undertaken to find solutions for this important interference problem. The first solution is based on the fact that a low-frequency rotating magnetic field generated for actuation purpose does not influence a high-frequency alternating magnetic field of the localization system. Exciting coil arrays for generating high-frequency alternating magnetic field are used in conjunction with detecting coil arrays to track a resonating coil enclosed inside the capsule ${ }^{14,15}$. The second idea is to place magnetic sensors inside the capsule to take advantage of the external magnetic field generated for actuation. Different approaches to measure this magnetic field have been proposed such as enclosing four or six one-axis Hall-effect sensors in the capsule ${ }^{16,17}$; using only one 3 -axis magnetic sensor ${ }^{18}$; or utilizing Hall-effect sensors combined with a tri-axial accelerometer ${ }^{19}$. A common drawback of these methods is space requirement to install such cumbersome localization 
mechanisms inside the capsule. In fact, the currently available capsules are already highly compact $^{20}$.

In this paper, we propose a novel localization method for WCE based on tracking multiple radioactive positron emission markers attached in the capsule body. Each marker has a spherical shape with a maximum diameter of $1 \mathrm{~mm}$. The markers are placed in the plastic cover of the capsule as shown in Fig. 3. This method hardly occupies any additional space inside the capsule. In addition, zero battery consumption is also an advantage of this localization method.

Tracking implanted positron emission markers has been studied by Xu et al. ${ }^{21}$ in order to track tumor motion during radiation therapy. In this paper, we extend this principle to the localization of WCEs. However, the relative distance between the markers in the capsule is closer than in the tumor which makes the clustering problem more challenging. Additionally, the movement range of the capsule is larger than that of the tumor. A new effective tracking algorithm has been developed to determine the positions of the three markers. After implementing the algorithm using simulation data, the position and orientation of the capsule can be calculated in real-time with sub-millimeter accuracy. The proposed localization method would be compatible with different actuation mechanisms.

\section{PRINCIPLE OF OPERATION AND SYSTEM DESIGN}

\section{II.A. Principle of operation}

In order to solve the localization problem for the WCE, three positron emission markers are embedded inside the capsule. The isotope confined in the marker decays and emits a positron. The emitted positron then encounters an electron from the environment. These two particles mutually annihilate and generate two gamma rays $(511 \mathrm{keV})$ in nearly collinear opposite directions. The distance that the positron has traveled before the annihilation event occurs is called positron range. Typically, it is within a few millimeters depending on the type of radioisotope. Consequently, in every localization run (50ms), hundreds (or thousands) of pairs of gamma rays in opposite directions are generated from the markers. The number of gamma rays generated is dependent on the activity of the particular isotope used. 


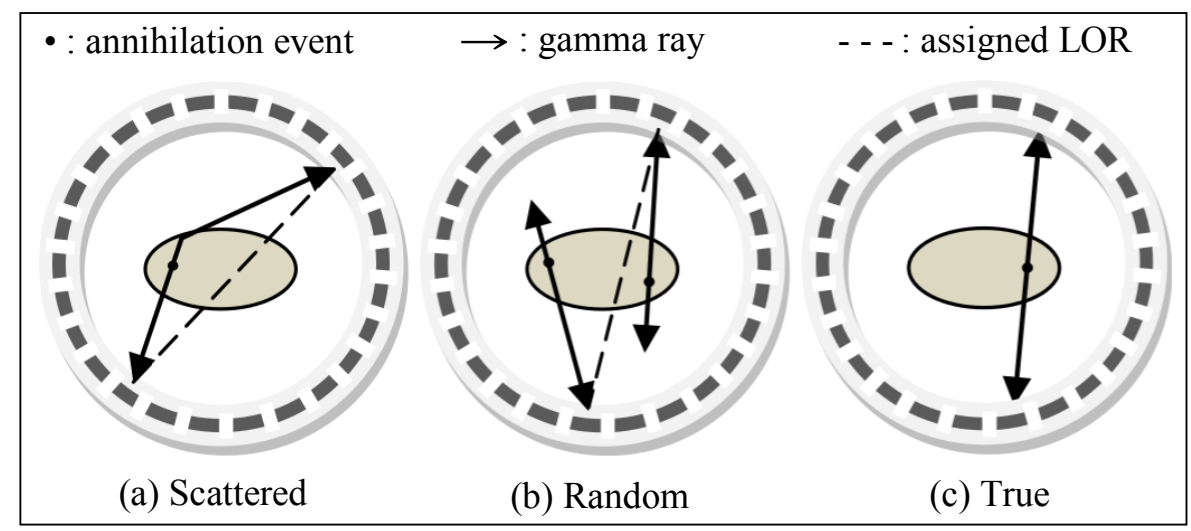

Fig. 1 Three main types of coincidence events.

Position-sensitive gamma ray detectors are placed around the patient to detect these annihilation gamma rays. Simultaneously detecting both annihilation photons defines a line of response (LOR), and this is called coincidence line. The location of the annihilation in the marker is supposed to be somewhere along this line. A coincidence event is considered to occur when two opposite detectors detect the gamma rays within a time window, a very short time interval (typically from six to twelve nanoseconds).

There are three major types of coincidence events: true, random, and scattered, as can be seen in Fig. 1. A scattered coincidence occurs when at least one of the gamma rays from a single annihilation event scatters in the object being scanned prior to detection. A random coincidence occurs when two gamma rays not arising from the same positron decay are detected in coincidence within a time window. Scattered and random coincidence events result in corrupted coincidence lines and less of spatial accuracy.

As mentioned above, a positron annihilation event is expected to have occurred somewhere along the coincidence line. Therefore, if there is only a single positron emission point source being scanned, ideally two non-parallel coincidence lines are sufficient to locate the source. However, in practice, due to the positron range, noncollinearity, random and scattered coincidences, it is necessary to have a sufficient number of coincidence lines to improve the tracking accuracy. In this case, the 3D location of the point source can be estimated by finding the point that minimizes the sum of squared perpendicular distances to all of the recorded coincidence lines ${ }^{21}$.

Extending the above principle, it is possible to track three positron emission markers using gamma ray detectors. In one localization run, three groups of coincidence lines originating 


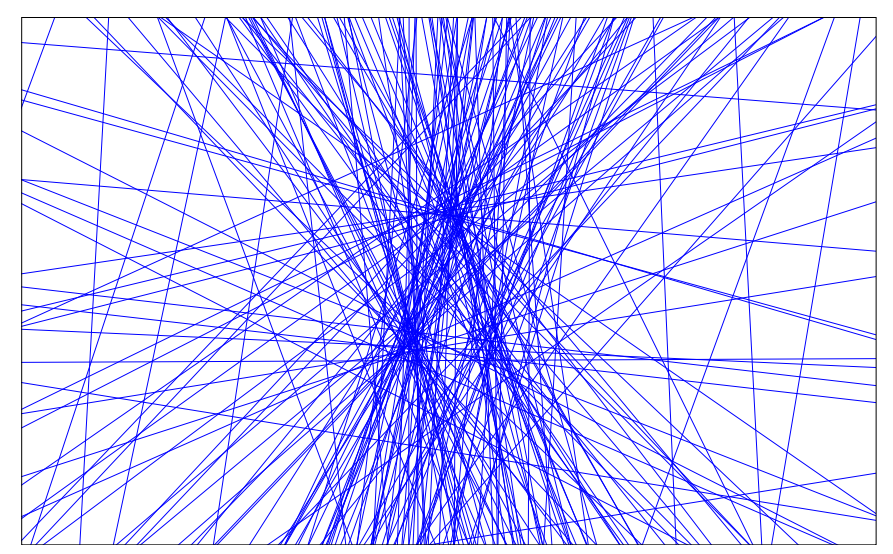

FIG. 2 Coincidence lines generated by three positron emission markers in one localization time interval.

from the three markers together with a number of corrupted lines are collected as illustrated in Fig. 2 as an example. In order to calculate the position of each marker, firstly, most of the corrupted lines are discarded by an outlier removal method. Then, a clustering algorithm is employed to assign correctly each coincidence line to the corresponding marker that has generated the coincidence line. Once this step is done, the position of each individual marker can be determined from its set of coincidence lines similar to the case of tracking a single point source. The position and orientation information of the capsule can then be obtained based on the position data of the three markers. These steps are explained in detail in the remainder of this paper.

\section{II.B. Conceptual system design}

- "Capsule with markers" prototype: For the tracking purpose, three cylindrical holes (with a dimension of $\Phi 1 \mathrm{~mm} \times L 1 \mathrm{~mm}$ ) are created in the plastic cover of the capsule body as can be seen in Fig. 3. At the beginning of an endoscopy procedure, three positron emission markers are attached to the capsule by inserting them into the holes. The marker will then be locked by a lid (with the same material of the plastic cover) at the top of the hole to ensure the marker will not be released during the endoscopy procedure. The relative distances between any two of the three markers are $10 \mathrm{~mm}, 13 \mathrm{~mm}$, and $13 \mathrm{~mm}$, respectively.

- Isotopes and markers: In tumor tracking ${ }^{21}$, the choice of isotope is limited due to the restriction in the isotope's half-life. The markers would remain in the tumor after completing 


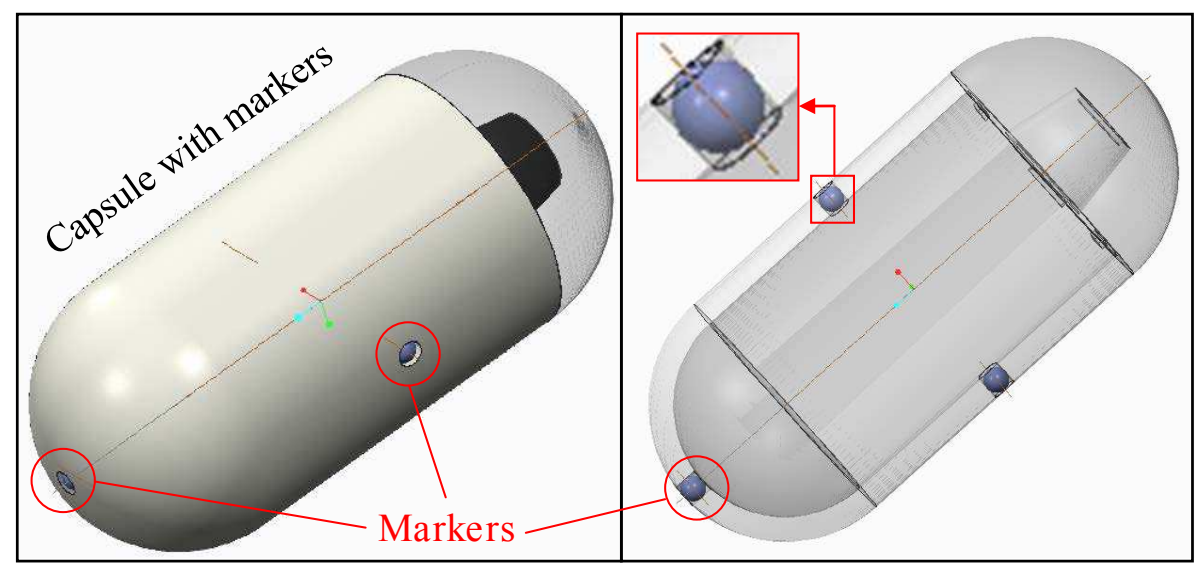

Fig. 3 Configuration of three markers in a capsule. The capsule is drawn transparently for better visualization of the markers (right). A hole confining the marker is shown and zoomed in a red box.

the radiation therapy, thus their half-lives are only allowed to be from a few days to a few weeks depending on the treatment duration ${ }^{21}$. This is to avoid high radiation dose to the patient. Conversely, in capsule localization, the capsule is generally disposed in normal excretion after traveling for approximately 8-10 hours inside the GI tract ${ }^{8}$. Therefore, in most cases, the patient will not be exposed to additional radiation once the WCE examination has been completed. Moreover, in the future when the capsule is provided with an active actuation system, the duration of a WCE examination is expected to even be shortened significantly and capsule retention is expected to not occur. Accordingly, this provides a broader range for choosing isotopes used in capsule tracking. Any positron emission isotope whose half-life is over a few hours could be a considerable choice.

In this study, we choose ${ }^{22} \mathrm{Na}$ (with a half-life of 2.6 years) to be the radioactive cores for the three markers. This is because ${ }^{22} \mathrm{Na}$ has already been used widely to make point sources for Positron Emission Tomography (PET) in medical clinics. Secondly, the positron range of ${ }^{22} \mathrm{Na}$ is smaller than that of other positron emission isotopes such as ${ }^{124} \mathrm{I},{ }^{74} \mathrm{As}$, ${ }^{84} \mathrm{Rb},{ }^{22}$ thus using ${ }^{22} \mathrm{Na}$ will potentially provide higher tracking accuracy. Finally, thanks to its long half-life, the markers can be re-used multiple times for future WCE examinations that require capsule localization without frequent replenishment.

The marker is designed to have a spherical shape with a diameter of less than $1 \mathrm{~mm}$. The marker contains a tiny spherical radioactive core ${ }^{22} \mathrm{Na}$ (with a diameter of $0.25 \mathrm{~mm}$ to $0.5 \mathrm{~mm}$, and an activity of less than $1.85 \mathrm{MBq}$ ) centered in an acrylic or metallic shell. 

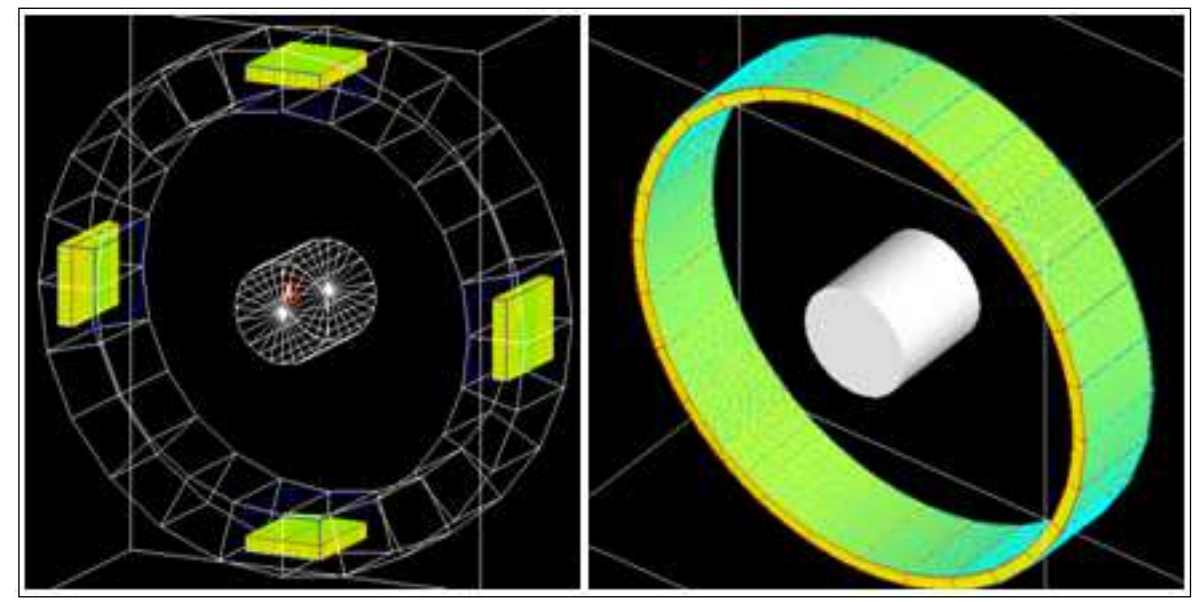

FIG. 4 Reduced geometry for capsule localization (left) compared to full-ring geometry for PET imaging (right).

For some other isotopes which have higher positron range such as ${ }^{124} I$, the spherical shell can be made by gold, tungsten or titanium with a thickness of $0.2-0.3 \mathrm{~mm}$ depending on the positron range of the isotopes ${ }^{22}$. This is to confine the emitted positrons to within the shell before annihilation occurs, and thus reduce the effect of positron range in the tracking accuracy. In practice, the marker design can be fabricated using similar techniques employed for producing commercial brachytherapy seeds ${ }^{21}$.

- Gamma ray detectors: Gamma ray detector modules which have been used in clinic PET imaging can also be employed in this tracking application for WCE. In conventional PET systems, for imaging purpose, the detectors need to be arranged in full rings to generate a number of images of the radioactive tracer distribution inside the patient body using reconstruction algorithms. However, for tracking purpose, in theory, a minimum of 2 pairs of facing detector blocks at a certain angle to each other is sufficient to provide the location information of the markers. This could significantly reduce the cost and the complexity of the localization system for WCE. Figure 4 shows a visual comparison between a conventional PET scanner and an example of detector modules for capsule localization.

In this paper, firstly, the performance of the localization method is evaluated using the full-ring geometry of a conventional PET system. Then, a smaller detector system with two pairs of detector modules is employed to analyze how reducing the detector geometry would affect the tracking accuracy. 


\section{II.C. Radiation dose estimation}

Since the physical half-life of ${ }^{22} \mathrm{Na}\left(T_{1 / 2}=2.6\right.$ years $)$ is much longer than the duration of one WCE examination (8-10 hours), the loss of activity due to the physical decay of the radionuclide can be neglected. The radiation dose from a marker to the surrounding tissues can thus be calculated by the external dose formula ${ }^{23}$ as below

$$
\dot{H}=\frac{\Gamma \times A \times e^{-\mu T}}{d^{2}}
$$

where $\dot{H}$ is the equivalent dose rate (in $\mu S v / h), \Gamma$ is the gamma constant of ${ }^{22} N a(0.362$ $\mu S v / h$ per $\mathrm{MBq} @ 1 \mathrm{~m}$ ), $A$ is the activity of the isotope (in $\mathrm{MBq}$ ), $\mu$ is the linear PET

attenuation coefficient $\left(\right.$ in $\left.\mathrm{cm}^{-1}\right)\left(\mu_{\text {tissues }}=0.096, \mu_{\text {bone }}=0.172^{24}\right)$, $\mathrm{T}$ is the thickness of the attenuating material (in $\mathrm{cm}$ ), and $d$ is the distance from the marker (in $\mathrm{m}$ ).

Therefore, the total radiation dose from one marker for an entire WCE examination is given by

$$
H=\int_{0}^{t} \dot{H} d t=\int_{0}^{t} \frac{\Gamma \times A \times e^{-\mu T}}{d^{2}} d t
$$

where $H$ is the total radiation dose in one WCE examination from one marker (in $\mu S v$ ) and $t$ is the duration of the examination (in hours). As the capsule moves during the WCE procedure, the distance $d$ from a tissue to the marker is a function of the time $d t$, the capsule velocity, and the structure of the GI tract. The architecture of the human GI tract, which consists of esophagus, stomach, small intestine and colon, is very complex, especially the small intestine. In addition, the capsule speed varies depending on a number of factors such as peristaltic propulsive force, the environment of the digestive section where the capsule is, an active control for capsule movement, etc. Therefore, it is impossible to calculate the exact radiation dose to a patient's tissues.

However, the maximum radiation dose to the surrounding tissues from the three markers can be approximately estimated by a simplified equation (when the capsule is assumed to remain stationary, and the gamma rays are assumed to pass through the air before reaching the tissue) as below

$$
H_{\max } \approx \frac{3 \times \Gamma \times A \times t}{d^{2}}
$$

As mentioned above, $A$ is chosen at $1.85 \mathrm{MBq}$, thus

$$
H_{\text {max }} \approx \frac{3 \times 0.362 \times 1.85 \times t}{d^{2}} \approx \frac{2.009 \times t}{d^{2}}(\mu S v)
$$




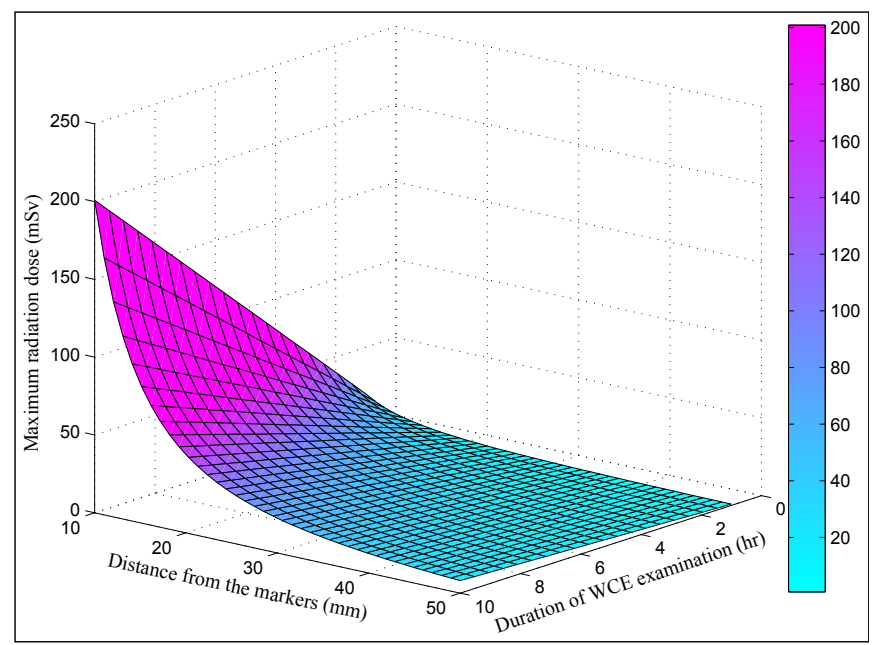

Fig. 5 Maximum radiation dose exposed from the three markers to surrounding tissues.

Figure 5 shows a distribution of the maximum radiation dose $(m S v)$ to surrounding tissues as a function of the duration of the WCE examination (from 1 to 10 hours) and the distance from the capsule (from $10 \mathrm{~mm}$ to $50 \mathrm{~mm}$ ). The further the distance from the capsule to the tissue is, the lower the radiation that it is exposed to. The radiation dose drops dramatically when the distance is larger than $20 \mathrm{~mm}$. The maximum radiation dose to a tissue that is $50 \mathrm{~mm}$ away from the capsule in 10 hours is approximately $8.04 \mathrm{mSv}$.

\section{TRACKING ALGORITHM}

As mentioned above, hundreds (or thousands) of coincidence lines are collected in every localization time interval $(50 \mathrm{~ms})$ which includes both true lines and corrupted ones. A tracking algorithm is required to extract position information of the three markers from the collected coincidence lines. The algorithm needs to perform fast enough to preserve realtime tracking while it ensures to provide high tracking accuracy. In this section, an effective tracking algorithm that we have developed based on Fuzzy C-means clustering algorithm is presented. This tracking algorithm is inspired by the algorithm proposed $i^{21,22,25}$. Our algorithm consists of four following main steps, and it can be extended to work with not only three markers, but also four, five or more if required.

1. An outlier removal method: to remove corrupted lines based on finding the center of the triangle formed by the three markers. This step provides clean input data for the 


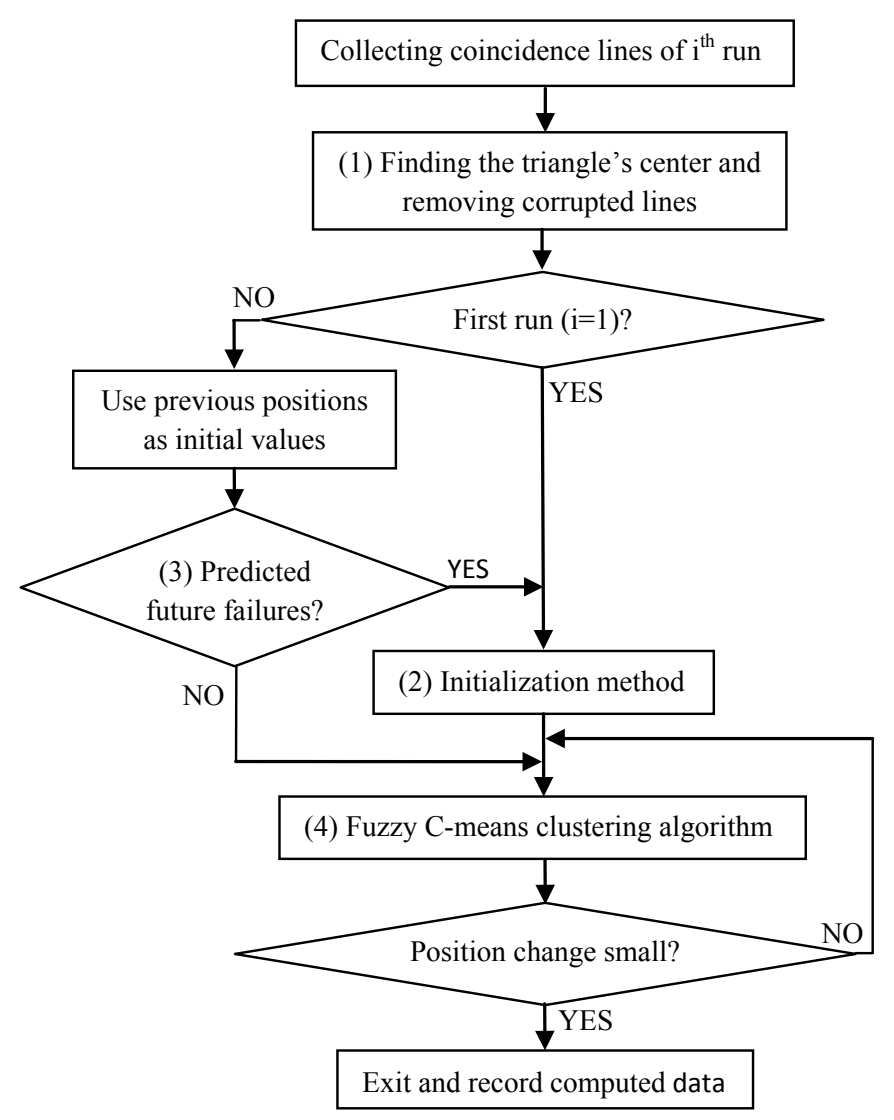

FIG. 6 Flow-chart of the tracking algorithm.

Fuzzy C-means clustering algorithm in step 4.

2. An initialization method: to estimate the initial points for the three vertices of the triangle (i.e. the initial positions of the three markers).

3. A failure prediction method: to ensure that the Fuzzy C-means clustering algorithm will not fail in some extreme cases using the initial values estimated in step 2.

4. The Fuzzy C-means clustering algorithm: to assign correctly each coincidence line to the corresponding marker that has generated it, and to calculate the point that minimizes the sum of squared perpendicular distances to all coincidence lines in each of the three classified groups.

The algorithm flow-chart is described in Fig. 6, and each step is explained in detail in the following sub-sections III.A to III.D 


\section{III.A. Removing corrupted lines and triangle center finding}

As shown in Fig. 3, the three markers form a triangle with the edge lengths of $10 \mathrm{~mm}$, $13 \mathrm{~mm}$ and $13 \mathrm{~mm}$, respectively. In this step, the center of the triangle is located. There are several types of triangle center, but the one that we are considering here is the triangle's centroid as it minimizes the sum of squared distances to the three vertices. This centroid is supposed to be the point that minimizes the sum of squared distances to all of the true coincidence lines.

In order to locate the center of the triangle, two following sub-steps are executed repeatedly in sequence until no coincidence lines are discarded and the change in the estimated position of the triangle center is less than a pre-determined value

- Finding the point that minimizes the sum of squared perpendicular distances to all coincidence lines (called minimum distance point).

- Removing coincidence lines that are too far from the calculated minimum distance point based on the modified Thompson Tau's method, a statistical outlier removal $\operatorname{method}^{26}$.

The mathematical formula representing iterations to determine the minimum distance point is given by

$$
\vec{C}^{(j+1)}=\vec{C}^{(j)}+\vec{T}^{(j)}
$$

where $\vec{C}^{(j+1)}$ is the new XYZ position of the center at the iteration $(j+1)^{t h} ; \vec{C}^{(j)}$ is the position of the center at the previous iteration $\left(j^{t h}\right)$. The initial position of the center $\vec{C}^{(j=0)}$ is set at $(0,0,0) \mathrm{mm}$ (which is the centroid of the detector system) for the first localization run $(i=1)$, and is set at the previously estimated position for other localization runs $(i>1)$. And $\vec{T}^{(j)}$ is a translation vector, defined by

$$
\vec{T}^{(j)}=k_{T} \frac{\sum_{n=1}^{N} G\left(\left\|\vec{d}\left(\vec{C}^{(j)}, l_{n}\right)\right\|, \bar{d}^{(j)}, \sigma^{(j)}\right) \cdot \vec{d}\left(\vec{C}^{(j)}, l_{n}\right)}{\sum_{n=1}^{N} G\left(\left\|\vec{d}\left(\vec{C}^{(j)}, l_{n}\right)\right\|, \bar{d}^{(j)}, \sigma^{(j)}\right)}
$$

where $\mathrm{N}$ is the number of coincidence lines collected in the current localization run $i^{\text {th }} ; k_{T}$ is a scale constant which influences the iteration speed (its optimal value is chosen at 0.5 ); 
$\vec{d}\left(\vec{C}^{(j)}, l_{n}\right)$ denotes the perpendicular distance vector from $\vec{C}(j)$ to a coincidence line $l_{n}$. Calculating distance vectors for every line $l_{n}(n=1, \ldots, N)$, we obtain a set $(S)$ of distance components with a mean $\bar{d}^{(j)}$ and a standard deviation $\sigma^{(j)}$. Each distance vector is then assigned a weight $G\left(\left\|\vec{d}\left(\vec{C}^{(j)}, l_{n}\right)\right\|, \bar{d}^{(j)}, \sigma^{(j)}\right)$ depending on how statistically inconsistent its magni-

tude is with the rest of the components in the set. The weight $G\left(\left\|\vec{d}\left(\vec{C}(j), l_{n}\right)\right\|, \bar{d}^{(j)}, \sigma^{(j)}\right)$ is computed according to the Gaussian probability distribution.

The iterations stop once the position change of the minimum distance point is less than a threshold $(0.1 \mathrm{~mm})$, i.e. when the magnitude of the translation vector $\vec{T}^{(j)}$ is less than $0.1 \mathrm{~mm}$.

The modified Thompson Tau's method ${ }^{26}$ is then activated to eliminate the coincidence lines that are two far from the calculated minimum distance point. With this method, only one corrupted line is rejected at a time. In each iteration step, the mean distance $\bar{d}$ and the standard deviation $\sigma$ of the set $(S)$ are re-calculated, and the line furthest from the minimum distance point is considered as a suspected corrupted line. Using the following rules, this line is determined to be rejected or to be kept

- If $\left(\left\|\vec{d}_{f}\right\|-\bar{d}\right)>\tau \cdot \sigma$, the line is discarded

- If $\left(\left\|\vec{d}_{f}\right\|-\bar{d}\right) \leq \tau \cdot \sigma$, the line is kept

where $\left\|\vec{d}_{f}\right\|$ is the distance from the minimum distance point to the furthest line. $\tau$ is the modified Thompson constant controlled by the number of coincidence lines in the set S. The value of $\tau$ can be selected from the table of modified Thompson $\tau$ values for different number of data points ${ }^{26}$. For example, $\tau$ varies from 1.9530 to 1.9572 when the number of data points is from 200 to 500 . Once a corrupted line is rejected, the process starts over and it is repeated until no more corrupted lines are identified.

\section{III.B. Initialization method}

Given a located triangle center, the purpose of this step is to estimate initial values for the three markers (i.e. the three vertices of the triangle). This can be done by rotating the triangle around its center such that the sum of squared distances from the vertices to their respective coincidence lines is minimal. Assume that the triangle is rotated by a rotation 
angle $\theta$ around a unit vector $(u, v, w)$ that goes through the triangle center, the positions of the three vertices are

$$
\left[\begin{array}{lll}
\vec{M}_{1} & \vec{M}_{2} & \vec{M}_{3}
\end{array}\right]=R(u, v, w, \theta) \cdot\left[\begin{array}{lll}
\vec{M}_{1}^{(0)} & \vec{M}_{2}^{(0)} & \vec{M}_{3}^{(0)}
\end{array}\right]+\left[\begin{array}{lll}
\vec{C} & \vec{C} & \vec{C}
\end{array}\right]
$$

where $\vec{C}$ is the location of the triangle center; $R(u, v, w, \theta)$ is a rotation matrix as a function of $(u, v, w, \theta)$; and $\left[\vec{M}_{1}^{(0)} \vec{M}_{2}^{(0)} \vec{M}_{3}^{(0)}\right]=\left[(-5,-4,0)^{T}(0,8,0)^{T}(5,-4,0)^{T}\right] \mathrm{mm}$ are reference positions of the three vertices when the triangle lies on the XY plane and the triangle center is placed at the origin of the Cartesian coordinate (i.e. the centroid of the detector system).

The sum of squared distances from the three vertices to their respective coincidence lines is a function of $(u, v, w, \theta)$ as below

$$
D(u, v, w, \theta)=\sum_{k=1}^{3} \sum_{j=1}^{N_{k}}\left\|\vec{d}\left(\vec{M}_{k}, l_{j}\right)\right\|^{2}
$$

where $\mathrm{k}$ is the marker index $(k=1,2,3) ; l_{j}$ is the coincidence line to which the marker $\vec{M}_{k}$ is the closest marker ( $l_{j}$ has a unit vector $\vec{n}_{j}$ and pass through a point $\left.\vec{a}_{j}\right) ; N_{k}$ is the number of coincidence lines to which $\vec{M}_{k}$ is the closest marker; $\left\|\vec{d}\left(\vec{M}_{k}, l_{j}\right)\right\|$ is the distance from the marker $\vec{M}_{k}$ to the line $l_{j}$.

To minimize $\mathrm{D}$, the parameters $(u, v, w, \theta)$ must satisfy the following equation

$$
\frac{\partial D}{\partial u}=\frac{\partial D}{\partial v}=\frac{\partial D}{\partial w}=\frac{\partial D}{\partial \theta}=0
$$

where $\quad c_{j}=\vec{a}_{j}^{T}\left[\vec{n}_{j}\right]_{\times}^{2} \vec{a}_{j}$

$$
\vec{V}_{j}^{T}=2 \vec{a}_{j}^{T}\left[\vec{n}_{j}\right]_{\times}^{2}
$$

0

$$
A_{j}=\left[\vec{n}_{j}\right]_{\times}^{2}
$$

Eq.(9) can thus be solved by a built-in optimization function in Matlab (e.g. fmincon). Once $(u, v, w, \theta)$ have been computed, the initial positions of the markers can be estimated using Eq.(7). This initialization method is generally time-consuming, thus it is only used when the localization starts or when a potential failure is detected. For other localization runs, the markers' positions calculated from the previous localization run are employed as initial data. 


\section{III.C. Fuzzy C-mean clustering algorithm}

Given the initial positions for the three markers estimated in step 2, Fuzzy C-mean

- The new positions of the markers and their relative activity are then updated:

$$
\vec{M}_{k}^{(i+1)}=\vec{M}_{k}^{(i)}+h \frac{\sum_{n=1}^{N}\left(u_{n k}^{(i)}\right)^{q} \cdot \vec{d}\left(\vec{M}_{k}^{(i)}, l_{n}\right)}{\sum_{n=1}^{N}\left(u_{n k}^{(i)}\right)^{q}}
$$


where $\mathrm{h}$ is a scale constant which controls the iteration speed (its optimal value is chosen at 1.5)

$$
f_{k}^{(i+1)}=\frac{\sum_{n=1}^{N} u_{n k}^{(i)}}{\sum_{j=1}^{3} \sum_{n=1}^{N} u_{n j}^{(i)}}
$$

As explained in the section III.A, most of the corrupted coincidence lines have been identified and discarded by removing coincidence lines that are too far from the triangle center. However, there may remain some coincidence lines passing close to the triangle center but not belonging to any clusters (their distances to each marker are larger than $5 \mathrm{~mm}$ ). Their assigned membership values would contribute to the new estimate of the markers' positions as shown in Eq.(16), and hence negatively affect the tracking accuracy. Furthermore, since the minimum distance between any two of the three markers are $10 \mathrm{~mm}$, a coincidence line whose distance to a marker $\vec{M}_{k}^{(i)}$ is larger than $5 \mathrm{~mm}$ is supposed to belong to other markers. Therefore, the following condition is added to Eq.(15)

$385 \quad u_{n k}^{(i)}=0$ if $\left\|\vec{d}\left(\vec{M}_{k}^{(i)}, l_{n}\right)\right\|>5 m m$

\section{III.D. Failure prediction method}

As explained above, the initialization method provides reliable initial values for the markers' positions which most likely lead to successful clustering by the Fuzzy C-mean algorithm. However, the computation of this initial estimate is cumbersome and time-consuming (approximately $0.4 \mathrm{~s}$ to $0.5 \mathrm{~s}$ ) compared to the sampling time of $50 \mathrm{~ms}$ (one localization time interval). The initialization method is thus only used for the first localization run when prior knowledge of the markers' positions is unknown. In other localization runs, markers' positions calculated from the previous run can be taken as initial positions for the markers.

In some extreme cases in which the capsule's position and orientation change dramatically after one localization run (e.g. when the patient coughs; or when the capsule falls in a hollow area such as a patient's stomach; or due to a sudden force generated by a magnetic actuation system), the initial estimate based on prior knowledge from the previous run may not be reliable. Wrong initial positions could result in a failure in the Fuzzy C-mean algorithm (the clustering algorithm is considered to fail when at least two markers are assigned to the same cluster). Therefore, a failure prediction method is essential to prevent the Fuzzy 
C-mean algorithm from having wrong starting values. Once the failure prediction method has detected a potential failure, the last known positions of the markers will not be used as the starting points for the clustering algorithm. Instead, the initialization method described in step 2 (sub-section III.B) is activated again to provide better initial data.

In order to avoid potential failures, two following conditions are checked before proceeding to the Fuzzy C-mean clustering algorithm:

- The relative distance between any two of the three initial points is compared with a pre-determined value $(5 \mathrm{~mm})$. If the former is smaller than the latter, the initial points are considered as unacceptable. This is because two initial points that are too close to each other would likely be assigned to the same cluster.

- If the capsule encounters an abrupt movement, the position change of the markers between two consecutive localization runs would be large. The collected coincidence lines would thus be far away from the three initial points. In order to check whether or not this condition occurs, the failure prediction method compares the total number of "close" lines to the three initial points with $50 \%$ of the total number of coincidence lines collected in the localization run. The initial positions are considered to be good only if the former is larger than the latter. A coincidence line is considered to be "close" with a marker if their perpendicular distance is less than $5 \mathrm{~mm}$.

\section{GATE SIMULATION}

In order to examine the validation of the proposed localization system, a model of the localization system has been simulated in GATE (Geant4 Application for Emission Tomography), a Monte Carlo simulation toolkit developed by the OpenGATE collaboration since $2001^{28}$. GATE is reliable to provide accurate modeling of Positron Emission Tomography (PET) and Single Photon Emission Tomography (SPECT). Many models of commercial PET and SPECT systems have been simulated in GATE and then validated by comparing the results generated by GATE against those obtained from the real systems. For example, the models of Allegro and Mosaic PET scanners from Philips ${ }^{29,30}$, GE Advance scanner from GEMS $^{31}$, and ECAT HRRT scanner from Siemens ${ }^{32}$ were validated with an agreement from $1 \%$ to $8 \%$. Thanks to the reliability, GATE toolkit has been used widely in many studies in 


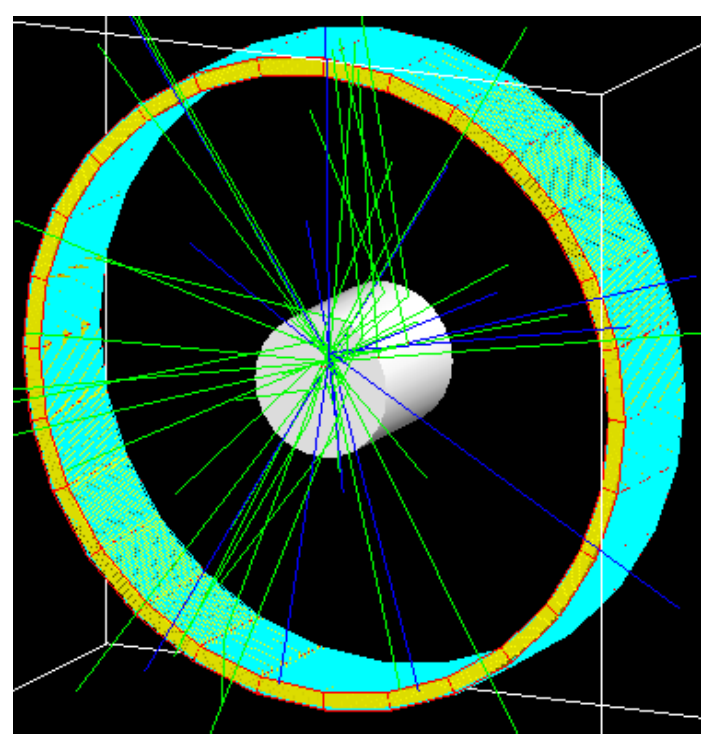

FIG. 7 Gamma rays are generated from a capsule inside a water phantom (white cylinder) in GATE simulation.

the field of nuclear medicine.

The model of the gamma ray detectors used in this study is designed according to the geometry of the Philips Allegro PET scanner ${ }^{29}$. A cylindrical water phantom with a diameter of $20 \mathrm{~cm}$ and a length of $20 \mathrm{~cm}$ is placed at the center of the scanner to simulate a patient body as illustrated in Fig. 7. The phantom is filled with water to maintain similar attenuation and scattering properties when the gamma rays pass through a patient body. A plastic capsule with a diameter of $11 \mathrm{~mm}$ and a length of $25 \mathrm{~mm}$ is placed inside the phantom. The thickness of the capsule's cover is $2 \mathrm{~mm}$. Three radioactive markers are embedded in the capsule's cover with the same configuration as shown in Fig. 3. The modeled marker contains $0.5 \mathrm{~mm}$-diameter spherical radioactive core encapsulated in a spherical acrylic shell with a thickness of $0.25 \mathrm{~mm}$. Isotope ${ }^{22} \mathrm{Na}$ with an activity of $1.85 \mathrm{MBq}$ is used as the radioactive core confined inside the markers.

The capsule is moved in an orbiting trajectory around the $\mathrm{z}$ axis with a speed of 5 $\mathrm{mm} / \mathrm{s}$, combined with a translation movement along the $\mathrm{z}$ axis with a speed of $2.5 \mathrm{~mm} / \mathrm{s}$ as illustrated in Fig. 8. This is to ensure that the positions of the markers change in all X, $\mathrm{Y}$ and $\mathrm{Z}$ directions during the movement of the capsule. Note that the orientation of the coordinate system in GATE is different with that of a regular Cartesian coordinate system. As can be seen in Fig. 8, x,y, and $\mathrm{z}$ axes in GATE are the lateral axis, vertical axis and 


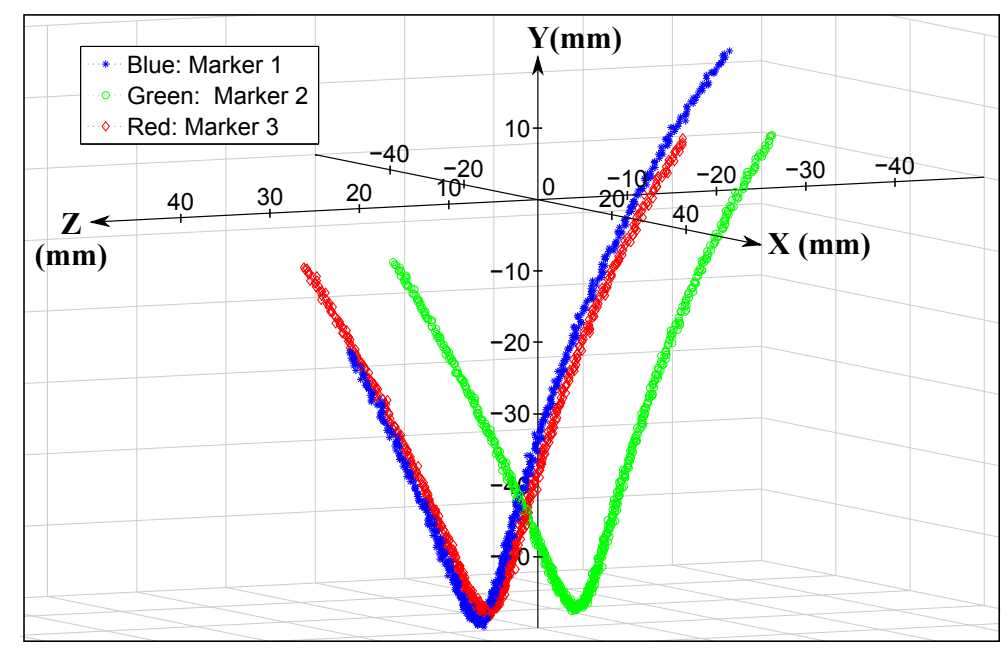

FiG. 8 Computed 3D positions of the three markers in all localization runs.

longitudinal axis of the detector system, respectively. The capsule travels from a starting point at the location of $(-57,0,-45) \mathrm{mm}$ to the stopping point at the location of $(57,0,45)$ $\mathrm{mm}$ in 36 seconds. Initially, the capsule is placed in a vertical direction, thus the positions of the three markers are $(-57,12,-45) \mathrm{mm},(-57,0,-40) \mathrm{mm}$, and $(-57,0,-50) \mathrm{mm}$, respectively. When the capsule finishes its journey, the markers' positions are $(57,-12,45) \mathrm{mm},(57,0$, 50) $\mathrm{mm}$, and $(57,0,40) \mathrm{mm}$, respectively.

Localization time interval is chosen at $50 \mathrm{~ms}$, i.e. coincidence lines are collected at a sampling frequency of $20 \mathrm{~Hz}$. Therefore, there are $36 \times 20=720$ localization runs throughout the simulation. In total, 1,735,600 coincidence lines are acquired. Accordingly, the average number of coincidence lines in each localization run is approximately 2,410 lines. In GATE, it is possible to obtain the number of random and scattered coincidence events after the simulation is completed by processing ROOT output files using $\mathrm{C}++$ codes. The total number of true coincidence events recorded is approximately 1,268,400 events, thus the fraction of true coincidence lines over the total number of coincidence lines is $73.1 \%$.

\section{RESULTS}

The tracking algorithm is implemented in Matlab (MathWorks, Inc.) to evaluate its performance using the data obtained by GATE. The localization is successful in all 720 localization runs. Figure 8 presents a plot of calculated positions of the three markers in 3D for every localization run which forms three corresponding trajectories. These trajectories 


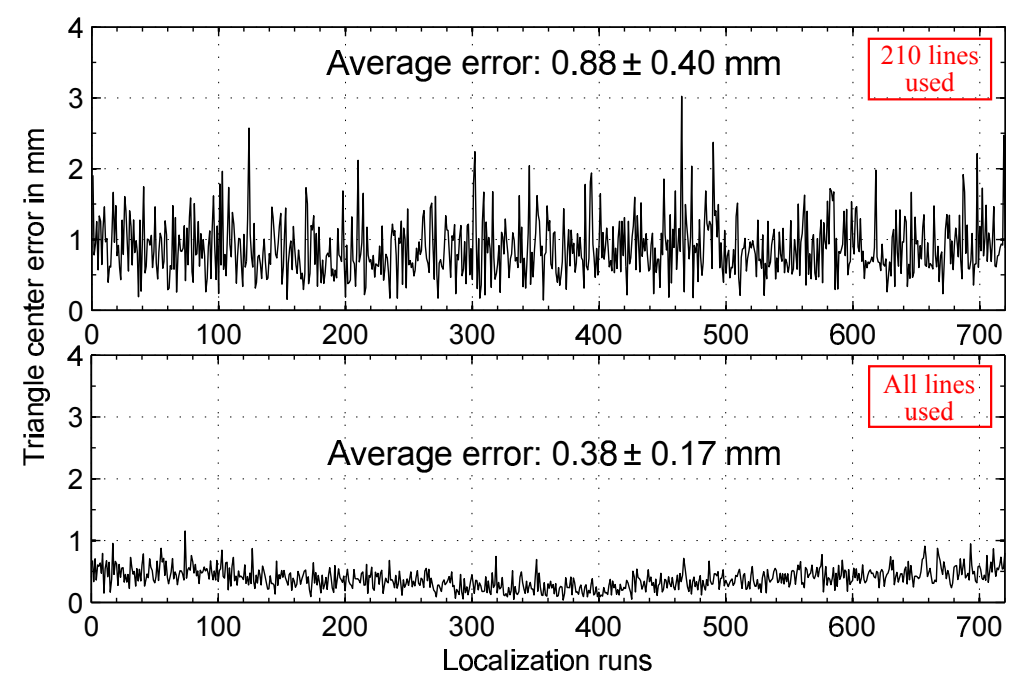

FIG. 9 The triangle center error in every localization run when only 210 lines (top) or all 2410 lines (bottom) are used in each localization time interval.

are closely related to the movement trajectory of the capsule. The localization performance is analyzed in detail in the sub-sections V.A to V.D

\section{V.A. Accuracy of finding triangle's center and effectiveness of removing corrupted lines}

Although an average of 2410 lines are stored in each localization run, only 210 lines are used in the implementation of the tracking algorithm. This number of lines is sufficient to provide a reasonable tracking accuracy while speeding up the computation. A more important reason is to imitate the loss of sensitivity in the case of using a smaller detector system (as shown in Fig. 4) in practice.

The tracking algorithm uses $210 \times 720=151,200$ coincidence lines throughout 720 localization runs. However, approximately 37,800 coincidence lines are detected as corrupted lines and thus are discarded by the algorithm. Therefore, an average of $75 \%$ coincidence lines remain after applying the corrupted line removal method explained in the sub-section III.A. This fraction is almost the same as the rate of $73.1 \%$ true coincidence lines reported in GATE. The triangle center finding method successfully locates the triangle center in every localization run with an average error of $0.88 \mathrm{~mm}$ when 210 lines are used. The error could be improved to $0.38 \mathrm{~mm}$ when all 2410 lines are taken into account as shown in Fig. 9. However, 

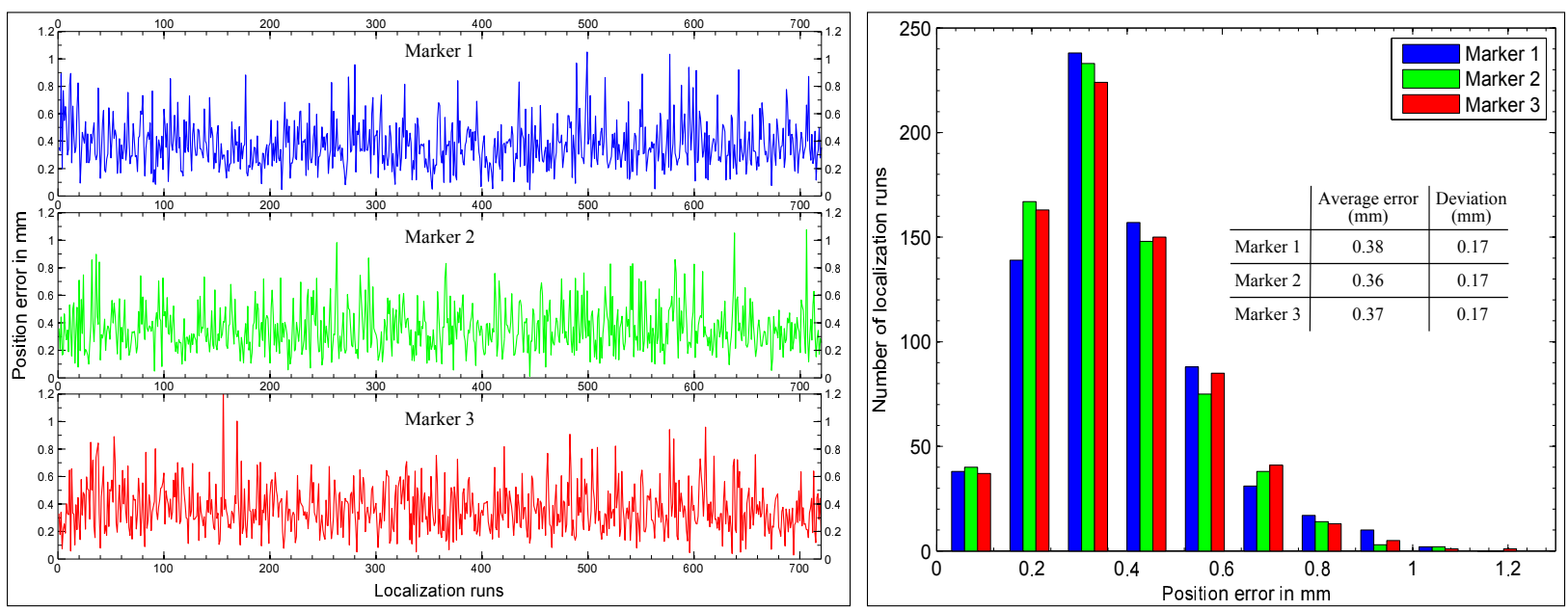

FIG. 10 Position error of each marker in every localization run (left) with a histogram of the markers' position errors throughout 720 localization runs (right).

the computational time is increased from $1.3 \mathrm{~ms}$ to $20.3 \mathrm{~ms}$ due to the large number of input data (computed by a $2.8 \mathrm{Ghz}$ Intel Core i7 processor).

\section{V.B. Performance of the tracking algorithm}

After the positions of the markers have been initialized by the initialization method in approximately $0.5 \mathrm{~s}$ for the first localization run, the Fuzzy C-mean clustering algorithm starts assigning the markers to their respective cluster of coincidence lines. For other localization runs, the prior knowledge of the markers' positions is used as initial points for the posterior run. With 210 coincidence lines (nearly 53 true coincidence lines for each marker) being used in each localization time interval, all three markers are localized successfully without any failures in every $50 \mathrm{~ms}$. Averagely, the whole tracking steps take $2.5 \mathrm{~ms}$ to complete locating the three markers in each localization run.

In GATE simulation, 3D locations of all annihilation events that occur during the capsule movement can be stored. The center of mass of these locations in each localization run is considered as the true position of the marker. This information is then compared with the estimated position computed by the tracking algorithm to evaluate the position error of each marker. As seen in Fig. 10, the tracking algorithm achieves high accuracy with an average position error of approximately $0.37 \mathrm{~mm}$.

Based on the configuration of the markers in the capsule body as shown in Fig. 3, the 


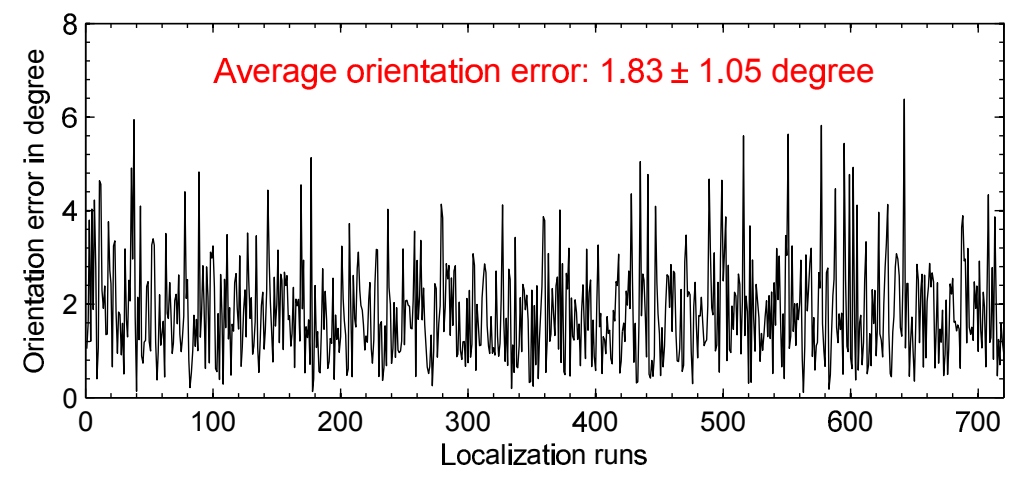

FIG. 11 Orientation error of the capsule in every localization run.

centroid of the capsule is also the midpoint of the segment connecting the two closest markers. The other marker, which is furthest away from these two markers, lies on one tip of the capsule. Therefore, a vector that originates from the midpoint between the two closest markers to the furthest marker can be considered as an orientation vector of the capsule. The true orientation vector can thus be calculated in every localization run using the true positions of the markers. The different angle between a true orientation vector and an estimated orientation vector is considered as the orientation error of the capsule. As illustrated in Fig. 11, the average orientation error of the capsule is approximately $1.8^{\circ}$.

\section{V.C. Failure prediction and initialization}

In order to evaluate the effectiveness of the failure prediction method, an abrupt movement of the capsule is generated at the beginning of the 300th localization run. The capsule is suddenly dragged $50 \mathrm{~mm}$ away from its previous position. Without the failure prediction method, the Fuzzy C-mean clustering algorithm fails to locate the three markers. This is understandable as the initial values, which are based on previous markers' positions, are unreliable in this case (50mm away). As a result, the markers' position errors are increased from an average of $0.4 \mathrm{~mm}$ to $50 \mathrm{~mm}$. Since the failure is not detected, wrong initial values are continuously fed into the tracking algorithm. The position errors, therefore, increase in each subsequent localization, as shown in Fig. 12.

At the beginning of the $500^{\text {th }}$ localization run, the failure prediction method is added to the tracking algorithm. This additional check immediately detects a potential failure and stops the use of prior data as initial values for the current localization run. The initialization 

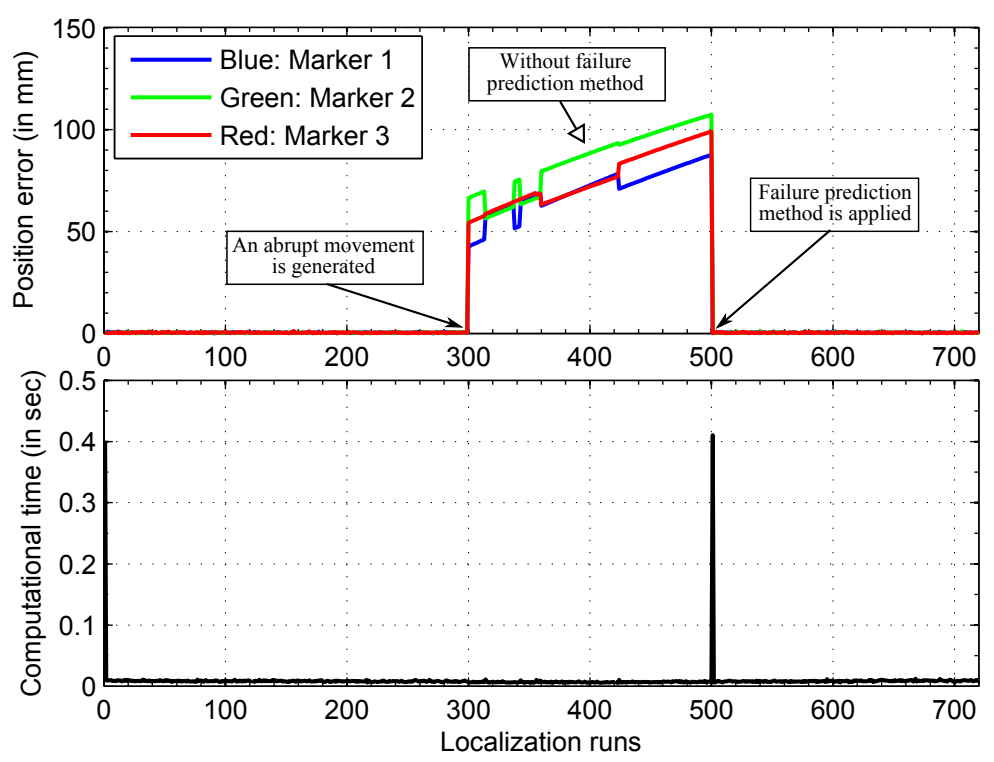

FIG. 12 Markers' position errors (top) and computational time (bottom) with and without the failure prediction method.

method is then activated to estimate new initial inputs for the Fuzzy C-mean clustering algorithm. This procedure takes 0.41s (shown as a surge on the bottom plot in Fig. 12) before the markers are kept on track with a similar accuracy as the first 300 runs. This demonstrates the necessity and effectiveness of the failure prediction method in maintaining a high accuracy for the localization procedure.

\section{V.D. Effect of phantom size and capsule movement speed on the tracking accuracy}

Scattering of gamma rays in a patient body affects the tracking accuracy. Scattering occurs more often in a thick patient body than in a smaller body, thus different dimensions of the phantom are simulated to evaluate the effect of a patient's thickness on the tracking performance. The phantom's diameters considered are $20 \mathrm{~cm}, 25 \mathrm{~cm}, 30 \mathrm{~cm}, 35 \mathrm{~cm}$, and 40 $\mathrm{cm}$. As can be seen in Fig. 13, the average position error of an entire simulation is higher when the phantom's diameter is increased. This is because the larger the diameter of the phantom is, the more scattered coincidence events occur, or in other words, the less true coincidence lines being obtained. Reported by GATE, an average percentage of true coincidence events in one entire simulation are $73.1 \%, 65.4 \%, 58.8 \%, 52.6 \%$, and $47.3 \%$ when 


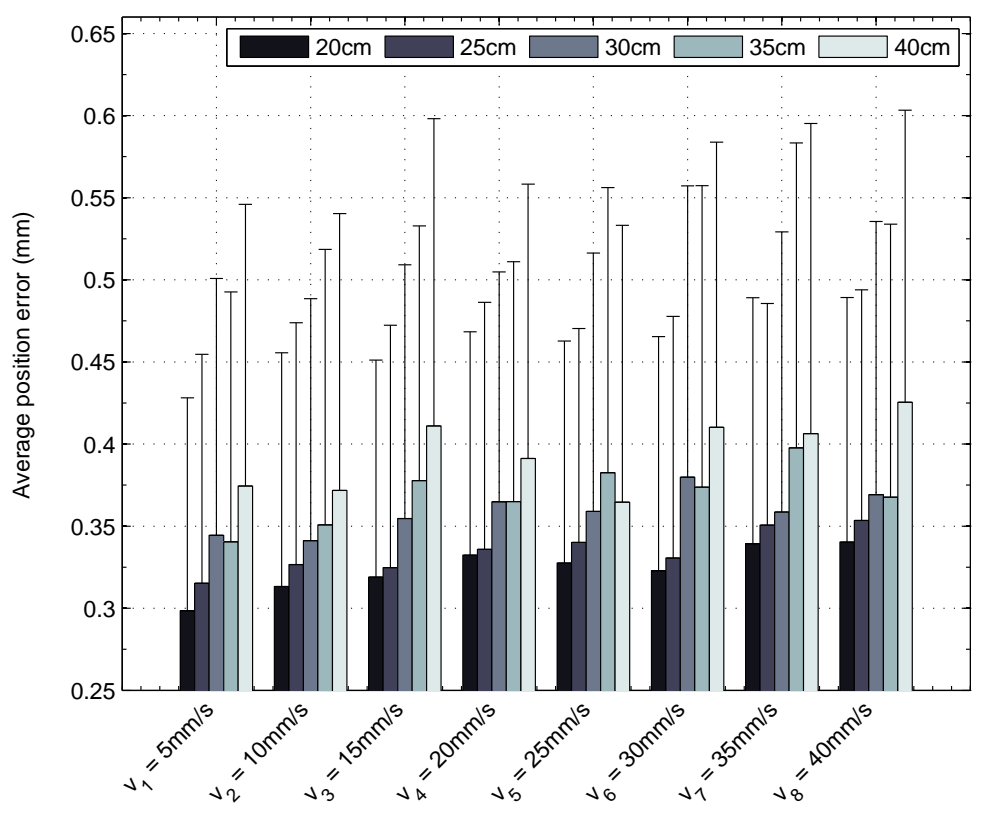

FIG. 13 Average position error of the capsule over an entire dataset versus movement speeds of the capsule and phantom sizes. Five bars in each group represent five different diameters of the phantom (from $20 \mathrm{~cm}$ to $40 \mathrm{~cm}$ ). One-side error bar is placed on the top of the main bar, which represents the standard deviation of the position error in one dataset.

the phantom's diameter varies from $20 \mathrm{~cm}$ to $40 \mathrm{~cm}$, respectively. Due to both attenuation and scattering characteristics of gamma rays, the total number of coincidence lines detected in each localization time interval is decreased in relation with the increase of the patient's thickness. Therefore, for comparison purposes the results shown in Fig. 13 are generated using all coincidence lines collected in one localization run instead of only 210 lines per run as previously.

In this section, effect of the movement speed of the capsule on the tracking performance is also investigated. The capsule moves along the same trajectory as previously, however eight different levels of the movement speeds are simulated, which are $5 \mathrm{~mm} / \mathrm{s}, 10 \mathrm{~mm} / \mathrm{s}, 15$ $\mathrm{mm} / \mathrm{s}, 20 \mathrm{~mm} / \mathrm{s}, 25 \mathrm{~mm} / \mathrm{s}, 30 \mathrm{~mm} / \mathrm{s}, 35 \mathrm{~mm} / \mathrm{s}$, and $40 \mathrm{~mm} / \mathrm{s}$ orbiting around the $\mathrm{z}$ axis, combined with $2.5 \mathrm{~mm} / \mathrm{s}, 5 \mathrm{~mm} / \mathrm{s}, 7.5 \mathrm{~mm} / \mathrm{s}, 10 \mathrm{~mm} / \mathrm{s}, 12.5 \mathrm{~mm} / \mathrm{s}, 15 \mathrm{~mm} / \mathrm{s}, 17.5 \mathrm{~mm} / \mathrm{s}$, and $20 \mathrm{~mm} / \mathrm{s}$ translation along the $\mathrm{z}$ axis, respectively. As shown in Fig. 13, the speed of the capsule movement has a slight effect on the tracking accuracy. The average position error becomes slightly higher when the capsule moves faster. In addition, the results have 

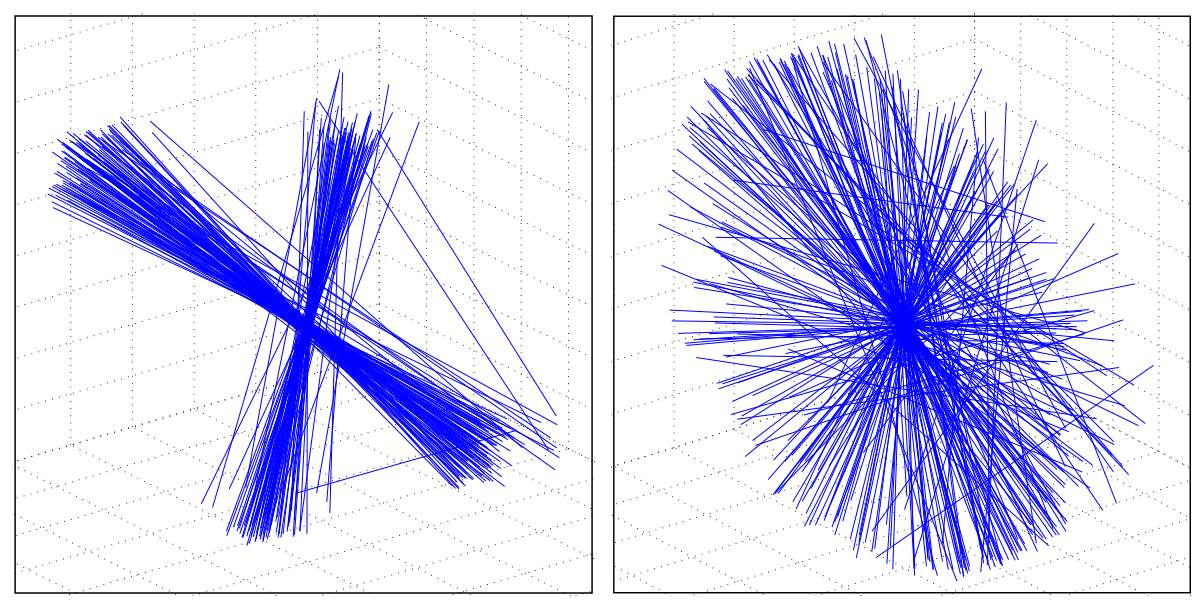

FIG. 14 Coincidence lines collected by a small detector system (left) and by the full-ring scanner (right).

demonstrated a high reliability of the tracking algorithm as three markers are successfully located in the worst case when the thickest patient body $(40 \mathrm{~cm})$ and the highest movement speed $(40 \mathrm{~mm} / \mathrm{s})$ are simulated.

\section{V.E. Performance comparison between the full-ring scanner and a smaller detector system}

As opposed to the conventional PET imaging technique, the localization method for WCE does not require the full-ring geometry. Therefore, a new simulation dataset, which is for a smaller detector system with two pairs of detector modules (Fig. 4), has been created to evaluate how a smaller detector geometry affects the tracking performance.

In order to compare these results with the results from the full-ring detector, most of the parameters of the previously simulated model are kept unchanged, such as the capsule speed $(5 \mathrm{~mm} / \mathrm{s})$, the capsule movement trajectory, the phantom size $(20 \mathrm{~cm})$, the crystal size

$(4 \mathrm{~mm} \times 6 \mathrm{~mm} \times 20 \mathrm{~mm})$, the crystal type (GSO), the detector diameter at the surface of the crystals $(86.4 \mathrm{~cm})$, and etc. However, instead of using 28 blocks (each block is made of $22 \times 29$ crystals), the new simulation uses only 4 blocks (each block contains $38 \times 29$ crystals). This means that the number of crystals used in this simulation is decreased by four times $(4 \times 38 \times 29=4,408$ crystals, compared with $28 \times 22 \times 29=17,864$ crystals $)$.

Due to the decreased number of the crystals, only 204,484 coincidence lines are recorded 
throughout 720 localization runs in this simulation (i.e. on average 284 lines per run). Again, 210 lines out of 284 lines are input to the tracking algorithm in each localization run to speed up the algorithm computation. Figure 14 illustrates a visual comparison between the coincidence lines collected in this simulation and those collected by the full-ring scanner. After the tracking algorithm is implemented, the tracking accuracy obtained is even enhanced slightly. The position errors of the three markers are $0.36 \mathrm{~mm} \pm 0.18 \mathrm{~mm}$, $0.36 \mathrm{~mm} \pm 0.19 \mathrm{~mm}$, and $0.35 \mathrm{~mm} \pm 0.17 \mathrm{~mm}$, respectively, and the success rate is maintained at $100 \%$.

TABLE I A comparison between the full-ring scanner and a small detector system in the number of total coincidence lines, the number of true coincidence lines, the true coincidence rate, and the average number of coincidence lines in each localization run when different phantom sizes are simulated

\begin{tabular}{|c|l|r|r|r|r|}
\hline $\begin{array}{c}\text { Phantom } \\
\text { diameters }\end{array}$ & $\begin{array}{c}\text { Detector } \\
\text { systems }\end{array}$ & $\begin{array}{r}\text { Number of } \\
\text { total lines }\end{array}$ & $\begin{array}{r}\text { Number of } \\
\text { true lines }\end{array}$ & $\begin{array}{c}\text { True coincidence } \\
\text { rate }\end{array}$ & $\begin{array}{r}\text { Average number } \\
\text { of lines per run }\end{array}$ \\
\hline \multirow{2}{*}{$20 \mathrm{~cm}$} & Full ring & $1,735,600$ & $1,268,390$ & $73.1 \%$ & 2410 \\
\cline { 2 - 6 } & Reduced & 204,484 & 167,512 & $81.9 \%$ & 284 \\
\hline \multirow{2}{*}{$25 \mathrm{~cm}$} & Full ring & $1,147,045$ & 750,291 & $65.4 \%$ & 1593 \\
\cline { 2 - 6 } & Reduced & 131,947 & 100,194 & $75.9 \%$ & 183 \\
\hline \multirow{2}{*}{$30 \mathrm{~cm}$} & Full ring & 768,010 & 451,483 & $58.8 \%$ & 1067 \\
\cline { 2 - 6 } & Reduced & 86,376 & 60,593 & $70.2 \%$ & 120 \\
\hline \multirow{2}{*}{$35 \mathrm{~cm}$} & Full ring & 519,842 & 273,639 & $52.6 \%$ & 722 \\
\cline { 2 - 6 } & Reduced & 57,360 & 36,809 & $64.2 \%$ & 80 \\
\hline \multirow{2}{*}{$40 \mathrm{~cm}$} & Full ring & 354,354 & 167,442 & $47.3 \%$ & 492 \\
\cline { 2 - 6 } & Reduced & 38,547 & 22,800 & $59.2 \%$ & 54 \\
\hline
\end{tabular}

There are two reasons for why the tracking accuracy is slightly better than previously achieved when a full-ring scanner is used. Firstly, this is because of the increase in the true coincidence rate (as shown in Table I). Given a less number of detector blocks (4 compared to 28), the possibility of detecting scattered coincidence and random coincidence by the smaller detector system is reduced. Secondly, regardless of how many coincidence lines are recorded totally in each localization run, the same number of lines (210) is fed to the tracking algorithm for both datasets. Therefore, the tracking algorithm was implemented with a larger number of true coincidence lines for this simulation dataset.

Although the activity of the isotope is chosen at the same level for both datasets, the success rate using the reduced geometry is retained at 100\%. However, with a larger phan- 


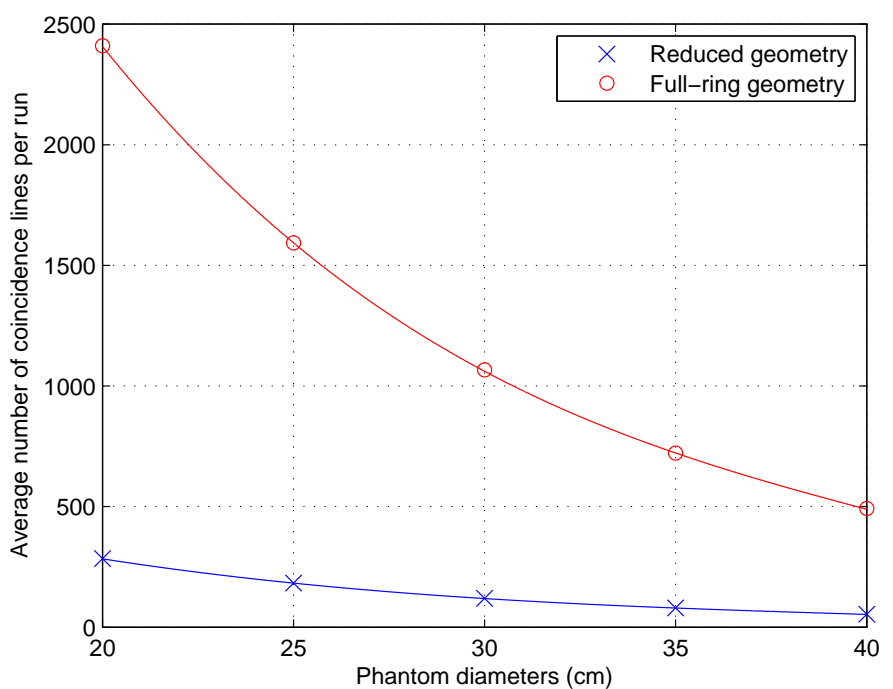

FIG. 15 Average number of coincidence lines collected in each localization run by both scanners when different phantom diameters are simulated. These data are fitted in two third-order polynomial curves colored correspondingly.

tom diameter, the average number of coincidence lines recorded in each localization run is expected to decrease to be less than the desired number of 210 . Therefore, to determine what activity of the isotope should be chosen when the patient thickness is changed, different phantom diameters have also been simulated. Table I presents a comparison in the number of coincidence lines and the true coincidence rate collected by both detector systems when different phantom diameters are used.

Depending on the tracking frequency and the phantom size, the minimum activity requirement of the isotope contained in the markers can approximately be estimated from:

$$
A_{\min }=1.85 \times \frac{f}{20} \times \frac{210}{n(d)}=\frac{19.425 \times f}{n(d)} \quad(\text { in } \mathrm{MBq})
$$

where $1.85 \mathrm{MBq}, 20 \mathrm{~Hz}, 210$ lines are the activity, the tracking frequency and the desired number of lines per run chosen in this study, $f$ is the tracking frequency, and $n(d)$ is the number of lines per run corresponding to the phantom diameter (d) that can be selected from the fitted curves as shown in Fig .15. 


\section{DISCUSSION}

One typical limitation of the conventional PET imaging technique is the field of view 605 (FOV) constraint, in which radioactive sources that fall beyond the FOV would not be imaged. Similarly, in this localization method, the capsule would not be localized once its movement exceeds the FOV. However, thanks to the real-time tracking (a tracking frequency of $20 \mathrm{~Hz}$ in this study), this limitation can be overcome. Being located in real-time, it is possible to ensure that the capsule is always within the FOV. When the capsule is close to the boundary of the FOV, the patient bed will be moved accordingly so that the current capsule position is approximately at the center of the FOV. In commercial PET systems, the patient bed position can be manipulated precisely in all lateral, vertical and longitudinal directions. The subsequent calculation to estimate the capsules localization information can then be compensated by adding the translation vector of the patient bed movement to the coordinates of the reference coordinate system.

The tracking algorithm developed in this study is reliable in computing the position and orientation of the capsule. Owing to the effectiveness of the failure prediction method and the initialization method based on finding the center of the triangle, the localization was never observed to fail over the datasets used in this study. Even in extreme cases where the capsule has an abrupt movement, the capsule can still be localized. The failure prediction method temporarily stops the use of the Fuzzy C-mean clustering algorithm in situations where initial positions of the markers are unacceptable, and waits until the initialization method is activated to provide more sufficient initial values to the clustering algorithm. In addition, given reasonably accurate estimated position of the triangle center (an average position error of $0.88 \mathrm{~mm}$ as shown in Fig. 9), the initialization method is likely to generate adequate initial values.

Due to safety and not causing discomfort to a patient, the capsule is not allowed to move too fast inside the GI tract. Additionally, abrupt capsule movement can be detected effectively by the failure prediction method. Therefore, the tracking frequency is not necessarily as high as $20 \mathrm{~Hz}$ performed in this study. With a lower tracking rate, i.e. longer sampling time, the same level of activity of the marker will provide a proportionally higher number of coincidence lines collected in each localization run. Accordingly, the activity of the marker can thus be chosen at a lower value. However, for thick and heavy patients, the activity 
would have to be increased to compensate the gamma rays that attenuate and scatter in

the patient body to ensure a sufficient number of coincidence lines are input to the tracking algorithm.

The localization method proposed in this paper is not limited to the application of robotic endoscopic capsules, and it can be extended to track other medical devices where position and orientation information is required in diagnosis and treatment of diseases. In such applications, the number of the markers embedded in the device can be varied (more than three) with various configurations, and they would be likely to be located by an algorithm similar to the tracking algorithm presented in this paper. In a future work, commercial PET scanners will be utilized to further validate the proposed localization method. Thanks to the high reliability of the GATE simulation which has been proved in the literature, the tracking performance is not expected to be much different when the method is evaluated with a real PET scanner.

\section{CONCLUSION}

This paper has presented an innovative localization method for WCE based on tracking three positron emission markers embedded in the capsule's cover. Using simulation data acquired from the GATE simulation toolkit, the performance of the tracking algorithm has been evaluated. Results obtained after implementing the tracking algorithm show that this localization method can potentially achieve real-time tracking with a high tracking accuracy. The position and orientation of the capsule can be computed in $2 \mathrm{~ms}$ to $3 \mathrm{~ms}$ (compared to $50 \mathrm{~ms}$ of sampling time) with an average position error of less than $0.4 \mathrm{~mm}$ and an average orientation error of less than $2^{\circ}$. Furthermore, free space occupation inside the capsule and zero battery consumption are also great advantages of this localization method. The localization system is expected to be compatible with any actuation mechanisms for WCE as there is no conflict between gamma rays and electric and magnetic fields used in other actuation systems. A disadvantage of this method is the radiation exposure to the patient body from the three radioactive markers. However, the activity used in this localization method (1.85MBq x 3 markers $=5.55 \mathrm{MBq}$ of ${ }^{22} \mathrm{Na}$ in several hours) is much lower than in clinical PET imaging (typically 200-600 MBq of ${ }^{18}$ F-FDG in approximately 2 hours ${ }^{33}$ ), and it can be lessened even more in practice if a lower tracking frequency is chosen. In future 
work, the tracking algorithm will be evaluated using experimental data when the localization

method is tested with conventional PET scanners.

\section{REFERENCES}

a) This work was supported in part by the funds available in Intelligent Nano-Tera Research Systems Laboratory

b) dtt581@uowmail.edu.au

c) gursel@uow.edu.au; corresponding author; ARC Centre of Excellence for Electromaterials Sience, University of Wollongong, New South Wales, 2522, Australia

d) steven.harvey@sesiahs.health.nsw.gov.au

e)hz467@uowmail.edu.au

f)weihuali@uow.edu.au

${ }^{1}$ G. Iddan, G. Meron, A. Glukhovsky, and P. Swain, "Wireless capsule endoscopy," Nature 405, 417-417 (2000).

${ }^{2}$ P. Swain, "At a watershed? technical developments in wireless capsule endoscopy," J. Dig. Dis 11, 259-265 (2010).

${ }^{3}$ B. Laulicht, N. J. Gidmark, A. Tripathi, and E. Mathiowitz, "Localization of magnetic pills," Proc. Natl. Acad. Sci. USA 108, 2252-2257 (2011).

${ }^{4}$ J. L. Gorlewicz, S. Battaglia, B. F. Smith, G. Ciuti, J. Gerding, A. Menciassi, K. L. Obstein, P. Valdastri, and R. J. Webster, "Wireless insufflation of the gastrointestinal tract," IEEE Trans. Biomed. Eng. 60, 1225-1233 (2013).

${ }^{5}$ S. P. Woods and T. G. Constandinou, "Wireless capsule endoscope for targeted drug delivery: Mechanics and design considerations," IEEE Trans. Biomed. Eng. 60, 945-953 (2013).

${ }^{6}$ K. Pahlavan, G. Bao, Y. Ye, S. Makarov, U. Khan, P. Swar, D. Cave, A. Karellas, P. Krishnamurthy, and K. Sayrafian, "Rf localization for wireless video capsule endoscopy," Int. J. Wireless Inform. Network 19, 326-340 (2012).

${ }^{7}$ K. Kong, D. Jeon, S. Yim, and S. Choi, "A robotic biopsy device for capsule endoscopy," J. Med. Devices 6, 031004-031012 (2012).

${ }^{8}$ J. L. Toennies, G. Tortora, M. Simi, P. Valdastri, and R. J. Webster, "Swallowable medical devices for diagnosis and surgery: the state of the art," Proc. Inst. Mech. Eng. C J. Mech. 
Eng. Sci. 224, 1397-1414 (2010).

${ }^{9}$ A. Arezzo, A. Menciassi, P. Valdastri, G. Ciuti, G. Lucarini, M. Salerno, C. Di Natali, M. Verra, P. Dario, and M. Morino, "Experimental assessment of a novel roboticallydriven endoscopic capsule compared to traditional colonoscopy," Dig. Liver Dis. 45, 657$662(2013)$.

${ }^{10}$ Y. Kusuda, "A further step beyond wireless capsule endoscopy," Sens. Rev 25, 259 - 260 (2005).

${ }^{11}$ T. D. Than, G. Alici, H. Zhou, and L. Weihua, "A review of localization systems for robotic endoscopic capsules," IEEE Trans. Biomed. Eng. 59, 2387-2399 (2012).

${ }^{12}$ D. Fischer, R. Schreiber, D. Levi, and R. Eliakim, "Capsule endoscopy: the localization system," Gastrointest. Endosc. Clin. N. Am. 14, 25-31 (2004).

${ }^{13}$ H. Chao, L. Mao, S. Shuang, Y. Wan'an, Z. Rui, and M. Q. H. Meng, "A cubic 3-axis magnetic sensor array for wirelessly tracking magnet position and orientation," IEEE Sens. J. 10, 903-913 (2010).

${ }^{14}$ S. Hashi, S. Yabukami, H. Kanetaka, K. Ishiyama, and K. I. Arai, "Wireless magnetic position-sensing system using optimized pickup coils for higher accuracy," IEEE Trans. Magn. 47, 3542-3545 (2011).

${ }^{15}$ I. Aoki, A. Uchiyama, K. Arai, K. Ishiyama, and S. Yabukami, "Detecting system of position and posture of capsule medical device," U. S. Patent US7815563 B2, 1-58 (2010).

${ }^{16}$ K. M. Miller, A. W. Mahoney, T. Schmid, and J. J. Abbott, "Proprioceptive magneticfield sensing for closed-loop control of magnetic capsule endoscopes," in 2012 IEEE/RSJ Int. Conf. IROS (2012) pp. 1994-1999.

${ }^{17}$ M.-G. Kim, Y.-S. Hong, and E.-J. Lim, "Position and orientation detection of capsule endoscopes in spiral motion," Int. J. Precis. Eng. Manuf. 11, 31-37 (2010).

${ }^{18}$ M. Salerno, G. Ciuti, G. Lucarini, R. Rizzo, P. Valdastri, A. Menciassi, A. Landi, and P. Dario, "A discrete-time localization method for capsule endoscopy based on on-board magnetic sensing," Meas. Sci. Technol 23, 015701-015710 (2012).

${ }^{19}$ C. Di Natali, M. Beccani, and P. Valdastri, "Real-time pose detection for magnetic medical devices," IEEE Trans. Magn. 49, 3524-3527 (2013).

${ }^{20}$ M. R. Basar, F. Malek, K. M. Juni, M. S. Idris, and M. I. M. Saleh, "Ingestible wireless capsule technology: A review of development and future indication," Int. J. Antenn. 
Propag 2012, 1-14 (2012).

${ }^{21}$ T. Xu, J. T. Wong, P. M. Shikhaliev, J. L. Ducote, M. S. Al-Ghazi, and S. Molloi, "Realtime tumor tracking using implanted positron emission markers: Concept and simulation study," Med. Phys. 33, 2598-2609 (2006).

${ }^{22}$ M. Chamberland, R. Wassenaar, B. Spencer, and T. Xu, "Performance evaluation of realtime motion tracking using positron emission fiducial markers," Med. Phys. 38, 810-819 (2011).

${ }^{23}$ H. Cember and T. Johnson, Introduction to Health Physics: Fourth Edition (Mcgraw-hill, 2008).

${ }^{24}$ J. H. Hubbell and S. M. Seltzer, "Tables of x-ray mass attenuation coefficients and mass energy-absorption coefficients from $1 \mathrm{kev}$ to $20 \mathrm{mev}$ for elements $\mathrm{z}=1$ to 92 and 48 additional substances of dosimetric interest," (1996).

${ }^{25}$ N. W. Churchill, M. Chamberland, and T. Xu, "Algorithm and simulation for real-time positron emission based tumor tracking using a linear fiducial marker," Med. Phys. 36, 1576-1586 (2009).

${ }^{26}$ A. Wheeler and A. Ganji, Introduction to Engineering Experimentation (Pearson Higher Education, 2010).

${ }^{27}$ J. C. Bezdek, Pattern Recognition with Fuzzy Objective Function Algorithms (Kluwer Academic Publishers, 1981).

${ }^{28}$ S. Jan, G. Santin, D. Strul, S. Staelens, K. Assi, D. Autret, S. Avner, R. Barbier, M. Bardis, P. M. Bloomfield, D. Brasse, V. Breton, P. Bruyndonckx, I. Buvat, A. F. Chatziioannou, Y. Choi, Y. H. Chung, C. Comtat, D. Donnarieix, L. Ferrer, S. J. Glick, C. J. Groiselle, D. Guez, P.-F. Honore, S. Kerhoas-Cavata, A. S. Kirov, V. Kohli, M. Koole, M. Krieguer, D. J. v. d. Laan, F. Lamare, G. Largeron, C. Lartizien, D. Lazaro, M. C. Maas, L. Maigne, F. Mayet, F. Melot, C. Merheb, E. Pennacchio, J. Perez, U. Pietrzyk, F. R. Rannou, M. Rey, D. R. Schaart, C. R. Schmidtlein, L. Simon, T. Y. Song, J.-M. Vieira, D. Visvikis, R. V. d. Walle, E. Wiers, and C. Morel, "Gate: a simulation toolkit for pet and spect," Phys. Med. Biol. 49, 4543 (2004).

${ }^{29}$ F. Lamare, A. Turzo, Y. Bizais, C. C. L. Rest, and D. Visvikis, "Validation of a monte 755 carlo simulation of the philips allegro/gemini pet systems using gate," Phys. Med. Biol. 51, $943(2006)$. 
${ }^{30}$ C. Merheb, S. Nicol, Y. Petegnief, J.-N. Talbot, and I. Buvat, "Assessment of the mosaic animal pet system response using list-mode data for validation of gate monte carlo modelling," Nucl. Instr. Meth. Phys. Res. A 569, 220-224 (2006).

${ }^{31}$ C. R. Schmidtlein, A. S. Kirov, S. A. Nehmeh, Y. E. Erdi, J. L. Humm, H. I. Amols, L. M. Bidaut, A. Ganin, C. W. Stearns, D. L. McDaniel, and K. A. Hamacher, "Validation of gate monte carlo simulations of the ge advance/discovery ls pet scanners," Med. Phys. 33, 198-208 (2006).

${ }^{32}$ F. Bataille, C. Comtat, S. Jan, and R. Trebossen, "Monte carlo simulation for the ecat 765 hrrt using gate," in 2004 IEEE NUCL. SCI. CONF. R, Vol. 4 (2004) pp. 2570-2574.

${ }^{33}$ C. Schiepers and M. Dahlbom, "Molecular imaging in oncology: the acceptance of pet/ct and the emergence of mr/pet imaging," Eur Radiol 21, 548-54 (2011). 